\section{Cyclophosphamide Exposure in Pediatric Systemic Lupus Erythematosus Is Associated with Reduced Serum Anti- Müllerian Hormone Levels}

\section{To the Editor:}

The reproductive risk to girls with pediatric systemic lupus erythematosus (pSLE) of varying disease severity and medication exposure is not well established. Because 15\%-20\% of patients with SLE are diagnosed before age 19 years ${ }^{1}$, many young women with pSLE will be affected by disease complications throughout their reproductive years. Although infertility in SLE has been attributed mainly to use of cyclophosphamide (CYC $)^{2,3}$, further studies are needed to clarify the relationship between disease severity, medication use, and ovarian dysfunction. Because the incidence of premature primary ovarian insufficiency (POI) after CYC exposure in patients with pSLE $<21$ years of age is estimated at $0-11 \%^{3}$, biomarkers for morbidities, such as infertility, are important because they may change current treatment strategies.

Anti-Müllerian hormone (AMH) is produced by granulosa cells of preantral and small antral follicles and has been identified as a sensitive biomarker of ovarian reserve. AMH concentrations are relatively stable throughout the menstrual cycle, are unaffected by hormonal contraceptives, and decline with advancing age, as does ovarian reserve/function ${ }^{4,5}$. Low to undetectable levels of AMH are found in women ages 25-46 years within 5 years of their final menstrual period, in cancer survivors exposed to chemotherapy and/or radiation therapy-induced follicle depletion, and in women with $\mathrm{POI}^{4,6,7}$.

To identify risk factors that may be associated with poor ovarian reserve and a risk of future infertility among patients with pSLE, we compared AMH levels among postmenarcheal young women with pSLE and a history of CYC exposure (pSLE+CYC; $n=6$; median age $19.2 \mathrm{yrs}$ ), women with pSLE and no history of CYC exposure (pSLE-CYC; $\mathrm{n}=17$; median age $19.5 \mathrm{yrs})$, and healthy age-matched controls $(\mathrm{n}=23$; median age $20.3 \mathrm{yrs}$ ). All subjects were Tanner stage $\mathrm{V}$ for breast and pubic hair development.

The clinical features of our patients with pSLE are summarized in Table 1. SLE was classified according to the revised 1997 American College of Rheumatology (ACR) criteria at $<19$ years of age. Disease activity and damage were assessed by the Systemic Lupus Erythematosus Disease Activity Index SELENA modification (SELENA-SLEDAI) and the Systemic Lupus International Collaborating Clinics (SLICC)/ACR Damage Index (SDI), respectively. Many patients were receiving prednisone (59\% pSLE-CYC and 100\% pSLE+CYC) and adjunctive immunosuppressive therapy ( $88 \%$ pSLE-CYC and 100\% pSLE+CYC), such as azathioprine and mycophenolate mofetil, at time of $\mathrm{AMH}$ measurement. Patients with pSLE and + CYC had received CYC 0.4-8.6 years prior to measuring AMH. No patient received gonadotrophin-releasing hormone $(\mathrm{GnRH})$ agonist cotherapy during $\mathrm{CYC}$ treatment. Assays for AMH were performed at Columbia University's Center for Women's Reproductive Care. Serum AMH levels (AMH Gen II ELISA; Beckman-Coulter) were determined in duplicate from blood samples taken irrespective of phase of menstrual cycle or use of oral contraceptives. The standard curve of this assay ranges from 0.05 to 10 $\mathrm{ng} / \mathrm{ml}$. Intra- and inter-assay coefficients of variation are $6.2 \%$ and $9.1 \%$, respectively.

Exposure to CYC in patients with pSLE is associated with a significant reduction in AMH (Figure 1A). The median AMH level in pSLE+CYC patients was lower than in pSLE-CYC patients and controls, $0.79 \mathrm{ng} / \mathrm{ml}$ versus $2.01 \mathrm{ng} / \mathrm{ml}$ or $1.92 \mathrm{ng} / \mathrm{ml}$, respectively $(\mathrm{p}=0.02)$. Median SLEDAI scores were similar (Figure 1B), but SDI scores were significantly higher in $\mathrm{pSLE}+\mathrm{CYC}$ patients $(\mathrm{p}=0.02$; Figure $1 \mathrm{C})$. Spearman rank correlation

Table 1. Pediatric systemic lupus erythematosus demographic and disease characteristics at the time of assessment of anti-Müllerian hormone (AMH).

\begin{tabular}{|c|c|c|c|c|c|c|c|c|c|}
\hline $\mathrm{Pt}$ & $\begin{array}{l}\text { Age, } \\
\text { yrs }\end{array}$ & $\begin{array}{c}\text { Disease } \\
\text { Duration, } \\
\text { yrs }\end{array}$ & $\begin{array}{c}\text { Regular } \\
\text { Menses, } \\
\text { Yes/No }\end{array}$ & SLEDAI & SDI & $\begin{array}{c}\text { Major SLE } \\
\text { Manifestations }\end{array}$ & $\begin{array}{c}\text { No. CYC Cycles/ } \\
\text { Total Dose } \\
\text { g }\end{array}$ & $\begin{array}{l}\text { Years } \\
\text { Since } \\
\text { CYC }\end{array}$ & $\begin{array}{l}\mathrm{AMH}, \\
\mathrm{ng} / \mathrm{ml}\end{array}$ \\
\hline 2 & 20 & 6.6 & $\mathrm{Y}$ & 30 & 2 & LN IV & & & 5.78 \\
\hline 3 & 16 & 0.5 & $\mathrm{Y}$ & 6 & 0 & & & & 2.62 \\
\hline 4 & 17 & 2.8 & $\mathrm{Y}$ & 8 & 0 & & & & 1.11 \\
\hline 7 & 21 & 2.1 & $\mathrm{~N}^{*}$ & 0 & 0 & LN III/V & & & 2.01 \\
\hline 8 & 18 & 9.8 & $\mathrm{Y}$ & 6 & 0 & LN IV/V, LN III/V, LSE & $9 / * *$ & 7.5 & 0.83 \\
\hline 9 & 24 & 9.7 & $\mathrm{Y}$ & 2 & 1 & LN IV, PHTN & $7 / * *$ & 8.6 & 0.75 \\
\hline 10 & 18 & 6.2 & $\mathrm{Y}$ & 20 & 2 & LN (no biopsy), CVA, NPSLE & $7 / 10$ & 3.5 & 0.22 \\
\hline 11 & 18 & 1.1 & $\mathrm{Y}$ & 7 & 0 & PLT & & & 1.65 \\
\hline 12 & 19 & 5.9 & $\mathrm{~N}$ & 4 & 0 & & & & 2.27 \\
\hline 17 & 18 & 8.3 & $\mathrm{Y}$ & 0 & 0 & & & & 1.84 \\
\hline 18 & 22 & 6.6 & $\mathrm{Y}$ & 2 & 0 & & & & 4.15 \\
\hline 19 & 19 & 7.5 & $\mathrm{~N}$ & 8 & 1 & LN III, DVT & & & 0.6 \\
\hline 20 & 15 & 8.1 & Y & 2 & 1 & LN V, DVT & & & 1.49 \\
\hline 21 & 17 & 2.6 & Y & 4 & 0 & & & & 1.94 \\
\hline 22 & 21 & 3.3 & $\mathrm{Y}$ & 4 & 0 & PLT & & & 2.15 \\
\hline 23 & 20 & 2.4 & Y & 0 & 0 & & & & 3.83 \\
\hline
\end{tabular}

* Taking Depo-Provera for contraception. ** Total cyclophosphamide (CYC) dosage could not be accurately calculated because CYC treatment started before transfer of care to our institution. CVA: cerebrovascular accident; DVT: deep vein thrombosis; LN: lupus nephritis; LSE: Libman-Sacks endocarditis; NPSLE: neuropsychiatric systemic lupus erythematosus; PHTN: pulmonary hypertension; PLT: significant thrombocytopenia; SLEDAI: Systemic Lupus Erythematosus Disease Activity Index; SDI: Systemic Lupus International Collaborating Clinics/American College of Rheumatology Damage Index 
A

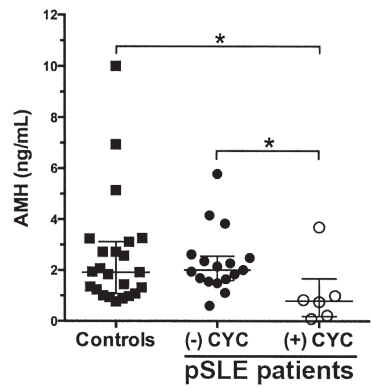

B

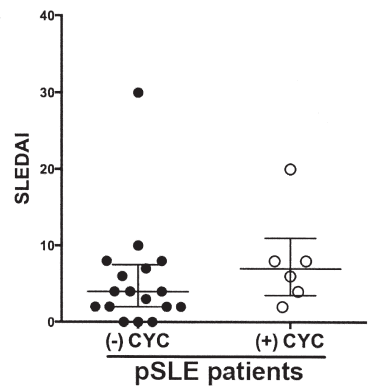

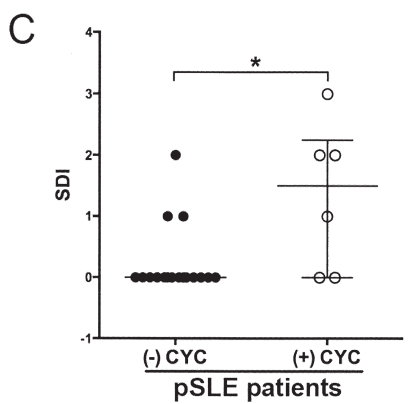

Figure 1. Comparison of AMH, SLEDAI, and SDI scores among patients with pSLE. A. Median serum AMH levels in healthy controls and pSLE-CYC patients were similar (1.92 ng/ml, IQR 1.08, $3.12 \mathrm{vs} 2.01$ $\mathrm{ng} / \mathrm{ml}$, IQR $1.60,2.55$, respectively). The median AMH level in $6 \mathrm{pSLE}+\mathrm{CYC}$ patients $(0.79 \mathrm{ng} / \mathrm{ml}, \mathrm{IQR}$ $0.19,1.67$ ) was significantly reduced compared to controls or pSLE-CYC patients. B. Median SLEDAI scores were similar in pSLE-CYC and pSLE+CYC patients, 4 (IQR 2, 7.5) versus 7 (IQR 3.5, 11). C. The median SDI score was significantly higher in pSLE+CYC patients, 1.5 (IQR 0,2.3) versus 0 . Medians, 25th percentile, and 75th percentile are shown. *Mann-Whitney U test, $p=0.02$. AMH: anti-Müllerian hormone; SLEDAI: Systemic Lupus Erythematosus Disease Activity Index; SDI: Systemic Lupus International Collaborating Clinics/American College of Rheumatology Damage Index; pSLE: pediatric systemic lupus erythematosus; CYC: cyclophosphamide; IQR: interquartile range.

indicated no association between AMH and SLEDAI $(r=-0.16, \mathrm{p}=0.47)$, $\mathrm{AMH}$ and SDI $(\mathrm{r}=-0.30, \mathrm{p}=0.16)$, and AMH and disease duration $(\mathrm{r}=$ $-0.27, \mathrm{p}=0.22$ ).

Reduced AMH levels in pSLE+CYC patients suggests impairment in ovarian reserve despite menstrual regularity, and raises concern regarding prognosis for their future fertility. Consistent with these findings, Aikawa, et al recently reported increased follicle-stimulating hormone and lower $\mathrm{AMH}$ in a similar cohort of pSLE patients treated with $\mathrm{CYC}^{8}$. It is encouraging that menstrual cycles and estradiol levels returned to normal in $75 \%$ of women $<35$ years of age with undetectable AMH levels after gonadotoxic chemotherapy ${ }^{7}$ and that low $\mathrm{AMH}$ in young healthy women ages 19-35 years did not predict reduced fecundability ${ }^{9}$. Oocyte quality of young women may allow for pregnancy despite reduced ovarian reserve (reflected by low AMH values). However, the significance of diminished $\mathrm{AMH}$ in women 15-25 years of age remains to be fully determined.

The effectiveness of GnRH agonist therapy for preservation of fertility in patients with pSLE who are undergoing chemotherapy has yet to be firmly established. However, Marder, et $a l^{10}$ recently showed AMH levels were higher in adult SLE patients who had received GnRH agonist cotreatment during CYC therapy. Such measures should be considered, because they may reduce risks of ovarian dysfunction in young women with severe disease manifestations. Although AMH levels increased by $0.5-0.8 \mathrm{ng} / \mathrm{ml}$ in a few patients who were 3 years post-CYC exposure ${ }^{5}$, the likelihood of AMH rebounding and the clinical significance of such increases in AMH post-CYC exposure remain unknown.

Our preliminary study is limited by the small number of patients from a single center and we lack ultrasound-derived antral follicle counts. Thus, our observations warrant confirmation with larger studies. Yet CYC is the standard of care treatment for severe manifestations of SLE, and therefore our observations should raise concern and encourage consideration of alternative therapies to $\mathrm{CYC}$, such as mycophenolate mofetil. We continue to follow our pSLE patients prospectively to observe trends in their AMH levels and to determine the relationship between AMH, SLE disease activity, and treatment regimens, as well as the future risk of infertility when AMH is reduced in this young cohort.

JOSEPHINE ISGRO, MD, Assistant Clinical Professor of Pediatrics, Pediatric Rheumatology, Morgan Stanley Children's Hospital of New York-Presbyterian, Columbia University Medical Center; SAHADAT K. NURUDEEN, MD, Clinical Fellow, Department of Obstetrics and Gynecology, Division of Reproductive Endocrinology and Infertility,
Columbia University College of Physicians and Surgeons; LISA F. IMUNDO, MD, Assistant Clinical Professor of Pediatrics, Pediatric Rheumatology, Morgan Stanley Children's Hospital of New York-Presbyterian, Columbia University Medical Center; MARK V. SAUER, MD, Professor of Obstetrics and Gynecology; NATAKI C. DOUGLAS, MD, PhD, Assistant Professor of Obstetrics and Gynecology, Division of Reproductive Endocrinology and Infertility, Columbia University College of Physicians and Surgeons, New York, New York, USA. Address correspondence to Dr. N.C. Douglas, Department of Obstetrics and Gynecology, Division of Reproductive Endocrinology and Infertility, New York Presbyterian Hospital-Columbia University Medical Center, 622 West 168th Street, PH 16-64, New York, NY 10032, USA. E-mail:nd2058@columbia.edu

\section{REFERENCES}

1. Levy DM, Kamphuis S. Systemic lupus erythematosus in children and adolescents. Pediatr Clin North Am 2012;59:345-64.

2. Hickman RA, Gordon C. Causes and management of infertility in systemic lupus erythematosus. Rheumatol 2011;50:1551-8.

3. Silva CA, Deen ME, Febronio MV, Oliveira SK, Terreri MT, Sacchetti SB, et al. Hormone profile in juvenile systemic lupus erythematosus with previous or current amenorrhea. Rheumatol Int 2011;31:1037-43.

4. Nelson SM, Anderson RA, Broekmans FJ, Raine-Fenning N, Fleming R, La Marca A. Anti-Mullerian hormone: clairvoyance or crystal clear? Hum Reprod 2012;27:631-6.

5. Clowse ME, Harward L, Criscione-Schreiber L, Pisetsky D, Copland S. Anti-Mullerian hormone: a better marker of ovarian damage from cyclophosphamide. Arthritis Rheum 2012;64:1305-10.

6. van Rooij IA, Broekmans FJ, Scheffer GJ, Looman CW, Habbema JD, de Jong FH, et al. Serum antimullerian hormone levels best reflect the reproductive decline with age in normal women with proven fertility: a longitudinal study. Fertil Steril 2005;83:979-87.

7. Yu B, Douglas N, Ferin MJ, Nakhuda GS, Crew K, Lobo RA, et al. Changes in markers of ovarian reserve and endocrine function in young women with breast cancer undergoing adjuvant chemotherapy. Cancer 2010;116:2099-105.

8. Aikawa NE, Sallum AM, Pereira RM, Suzuki L, Viana VS, Bonfa E, et al. Subclinical impairment of ovarian reserve in juvenile systemic lupus erythematosus after cyclophosphamide therapy. Clin Exp Rheumatol 2012;30:445-9. 
9. Hagen CP, Vestergaard S, Juul A, Skakkebaek NE, Andersson AM, Main KM, et al. Low concentration of circulating antimullerian hormone is not predictive of reduced fecundability in young healthy women: a prospective cohort study. Fertil Steril 2012;98:1602-8.

10. Marder W, McCune WJ, Wang L, Wing JJ, Fisseha S, McConnell DS, et al. Adjunctive GnRH - a treatment attenuates depletion of ovarian reserve associated with cyclophosphamide therapy in premenopausal SLE patients. Gynecol Endocrinol 2012;28:624-7.

J Rheumatol 2013;40:6; doi:10.3899/jrheum.130017 